\title{
Simulation and Characterization of a Miniaturized Scanning Electron Microscope
}

\author{
Jessica. A. Gaskin, Gregory A. Jerman \\ NASA Marshall Space Flight Center, Huntsville, AL 35812, Jessica.Gaskin@ nasa.gov, 256-961-7818 \\ Stephanie Medley, Don Gregory, Terry O. Abbott \\ University of Alabama in Huntsville, Huntsville, AL 35899 \\ Allen R. Sampson, \\ Advanced Research Systems, St. Charles, IL 60174
}

\begin{abstract}
A miniaturized Scanning Electron Microscope (mSEM) for in-situ lunar investigations is being developed at NASA Marshall Space Flight Center with colleagues from the University of Alabama in Huntsville (UAH), Advanced Research Systems (ARS), the University of Tennessee in Knoxville (UTK) and Case Western Reserve University (CWRU). This effort focuses on the characterization of individual components of the mSEM and simulation of the complete system. SEMs can provide information on the size, shape, morphology and chemical composition of lunar regolith. Understanding these basic properties will allow us to better estimate the challenges associated with In-Situ Resource Utilization and to improve our basic science knowledge of the lunar surface (either precluding the need for sample return or allowing differentiation of unique samples to be returned to Earth.) The main components of the mSEM prototype includes: a cold field emission electron gun (CFEG), focusing lens, deflection/scanning system and backscatter electron detector. Of these, the electron gun development is of particular importance as it dictates much of the design of the remaining components. A CFEG was chosen for use with the lunar MSEM as its emission does not depend on heating of the tungsten emitter (lower power), it offers a long operation lifetime, is orders of magnitude brighter than tungsten hairpin guns, has a small source size and exhibits low beam energy spread. ${ }^{1,2}$
\end{abstract}

\section{TABLE OF CONTENTS}

1. INTRODUCTION

2. Thermionic Pointed Filament (PF)

3. Cold Field EMisSion GUN

4. ConClusions

REFERENCES

BIOGRAPHIES

ACKNOWLEDGMENTS

\section{INTRODUCTION}

Scanning Electron Microscopes (SEMs) have become a mainstay tool for laboratories across the country. Capable of nano-scale imaging, with coincident chemical analysis (via Energy Dispersive Spectroscopy), SEMs are capable of

\footnotetext{
${ }^{1}$ 978-1-4244-7351-9/11/\$26.00 @2011 IEEE

${ }^{2}$ IEEEAC paper \#1594, Version 1, Updated October 26, 2010
}

supporting analyses across disciplines. Unfortunately, SEMs are rather large (desk size + supporting vacuum system). There are smaller desk-top versions, however; these are still not very portable. The group at NASA Marshall Space Flight Center, and colleagues, have designed and partially fabricated a portable - miniaturized SEM for use on the lunar surface. The concept of which can be adapted for multiple environments. The science justification for a lunar SEM has been reported on by our colleagues at UTK [1], [2].

The current mini-SEM prototype consists of a cold field emission electron gun (CFEG), followed by an electron focusing column and a custom scanning/magnification system. However, due to the nature of the CFEG and to maximize our effort, the development has been split into two parallel tracks.

The first track utilizes a thermionic pointed tungsten filament coupled to the electron focusing column and scanning system. This system requires a less stringent vacuum system and allows for several of the components to be characterized independently. The second track concerns the CFEG characterization. The CFEG requires an Ultra High Vacuum (ideally, low $10^{-10}$ Torr) for operation and a custom high voltage power supply to control the gun emission current. Once both systems are fully characterized, the CFEG will be attached to the electron column and scanning system and tested as a single unit.

\section{Thermionic Pointed Filament (PF)}

One stage of the development of the mini-SEM involves the use of a thermionic pointed filament attached to the electron focusing column and scanning system. A thermionic filament is desirable for several reasons. Mainly, it requires a high vacuum $\left(\sim 10^{-7}\right.$ Torr), but not ultra high for operation; it is not very expensive (around a tenth of the cost of a cold field emitter), and it is fairly robust. The negatives are: it is not as bright as a cold field emitter; it generally has a larger source size (30 - 100 $\mu \mathrm{m}$ versus $5 \mathrm{~nm}$ for a CFE), and the lifetime is shorter by a factor of 10 compared to a CFE [3].

For testing the electron focusing column and scanning system however, the thermionic emitter is ideal in that its operation is well known and it can be controlled using a 
readily available off-the-shelf power supply. To overcome some of the problems associated with the large source size of this type emitter, a pointed filament (Type PF), from EBSciences [4] was chosen. This pointed filament has been etched to have a $100 \mathrm{~nm}$ tip radius. A photo is shown in Figure 1 along with the standard design.
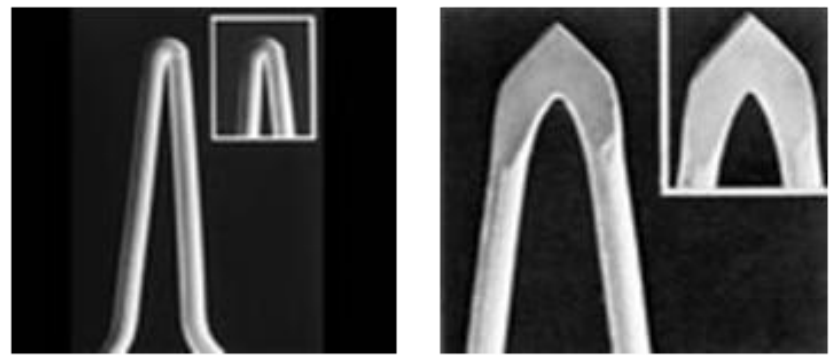

Figure 1. Comparison of a standard filament (left) with the EBSciences etched design used in this work [4].

The power supply used to operate this filament and the Wehnelt is commercially available (CPS inc. Model 6001 or higher, $30 \mathrm{kV}$ ) and the power supply for the secondary electrostatic lens is an off-the-shelf Bertram $380 \mathrm{X}( \pm 10 \mathrm{kV})$.

The thermionic gun configuration consists of a cathode, followed by a Wehnelt Cylinder and grounded anode. By applying a potential to the Wehnelt Cylinder, the electron emission is either suppressed or allowed to flow [3]. The following simulation and tests illustrate this point. The grounded anode acts to accelerate the emitted electrons which are at a high negative potential.

\section{PF Simulations}

The governing equation for the thermionic gun (Te-gun) is the Richard-Dushman equation,

$$
\mathrm{J}=\lambda_{\mathrm{R}} \mathrm{A} \mathrm{T}^{2} \exp [-\mathrm{W} / \mathrm{kT}]
$$

where $\lambda_{R}$ is a cathode material dependent constant about equal to 0.5 and the constant $\mathrm{A}$ is comprised of fundamental constants which combine to have a value of roughly 120 Amps $\mathrm{cm}^{-2} \mathrm{~K}^{-2}$ [5]. $\mathrm{T}$ is the temperature of the cathode, $\mathrm{W}$ is the work function of the cathode material, and $\mathrm{k}$ is Boltzman's constant. These constants $\left(\lambda_{R} \&\right.$ A $)$ are multiplied together into a constant also called " $A$ " in the Charged Particle Optics (CPO2DS) simulation software

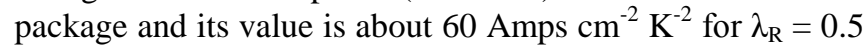
[6]. The $\mathrm{T}^{2}$ dependence is purely classical blackbody with the electrical power emitted (in terms of current density) being set equal to the $\mathrm{T}^{4}$ dependent Stefan-Boltzmann equation. The rest of the expression is the Planck probability density function.

Electrons are ejected from the cathode of diameter "CD" at high temperature and accelerated by the cathode voltage $\left(\mathrm{V}_{\text {cathode }}\right)$ toward the anode (at ground) which is a distance "d" away. A small fraction of the total number of electrons ejected passes through the aperture of diameter "DA" in the anode to be further directed by the electron optics or detected by a Faraday cup. The temperature is an adjustable parameter in the simulation and cannot be easily measured experimentally, but reasonable values can be estimated. A representative example of parameters used in a simulation of the thermionic electron gun that resulted in a current of 9.93 $\mu \mathrm{A}$ being emitted from the cathode and a current through the anode of $4.7 \mu \mathrm{A}$ is given in Table 1 . This pair of predicted values is reasonable as will be seen in the experimental results. The parameters in the table are not unique but they were chosen based on the actual design of the Te-gun.

Table 1. Parameters used in CPO2DS software simulation of the thermionic electron gun.

\begin{tabular}{|l|c|}
\hline & Thermionic electron gun \\
\hline $\mathrm{CD}(\mu \mathrm{m})$ & 0.1 \\
\hline $\mathrm{d}(\mathrm{cm})$ & 1 \\
\hline $\mathrm{DA}(\mu \mathrm{m})$ & 200 \\
\hline $\mathrm{V}_{\text {cathode }}(\mathrm{V})$ & -10000 \\
\hline $\mathrm{V}_{\text {anode }}(\mathrm{V})$ & 0 \\
\hline $\mathrm{W}(\mathrm{eV})$ & 4.5 \\
\hline $\mathrm{A}\left(\mathrm{Amp} / \mathrm{cm}^{2} / \mathrm{T}^{2}\right)$ & 60 \\
\hline $\mathrm{T}(\mathrm{K})$ & 2500 \\
\hline
\end{tabular}

Figures 2 and 3 illustrate the electron gun with cathode, Wehnelt Cylinder and anode. The effect of the Wehnelt voltage is apparent when the two figures are compared. In Figure 2, the Wehnelt Cylinder voltage is more positive than the cathode and in Figure 3, the Wehnelt Cylinder voltage is slightly more negative than the cathode. The lensing effect is obvious with the second case producing a more concentrated beam of electrons to (and through) the anode.

Two variations of the thermionic electron gun without the Wehnelt Cylinder were simulated: $d=1 \mathrm{~mm}$ and $\mathrm{d}=10 \mathrm{~mm}$. The current through the anode for the $d=10 \mathrm{~mm}$ case was found to be approximately half the current emitted by the cathode. The current through the anode for the $\mathrm{d}=1 \mathrm{~mm}$ case was approximately equal to the cathode current.

The CPO software employs segments to define rays; each ray representing a bundle of electrons. For the thermal emission gun case the segments were chosen to be equal sizes (widths) and equally spaced, but not equal areas. Parameters were chosen based on the assumption of cylindrical symmetry. When the distribution of the segments was varied, the current emitted was mostly unaffected as long as the emission areas were held constant. A change in the number of segments did not significantly affect the current emitted. 


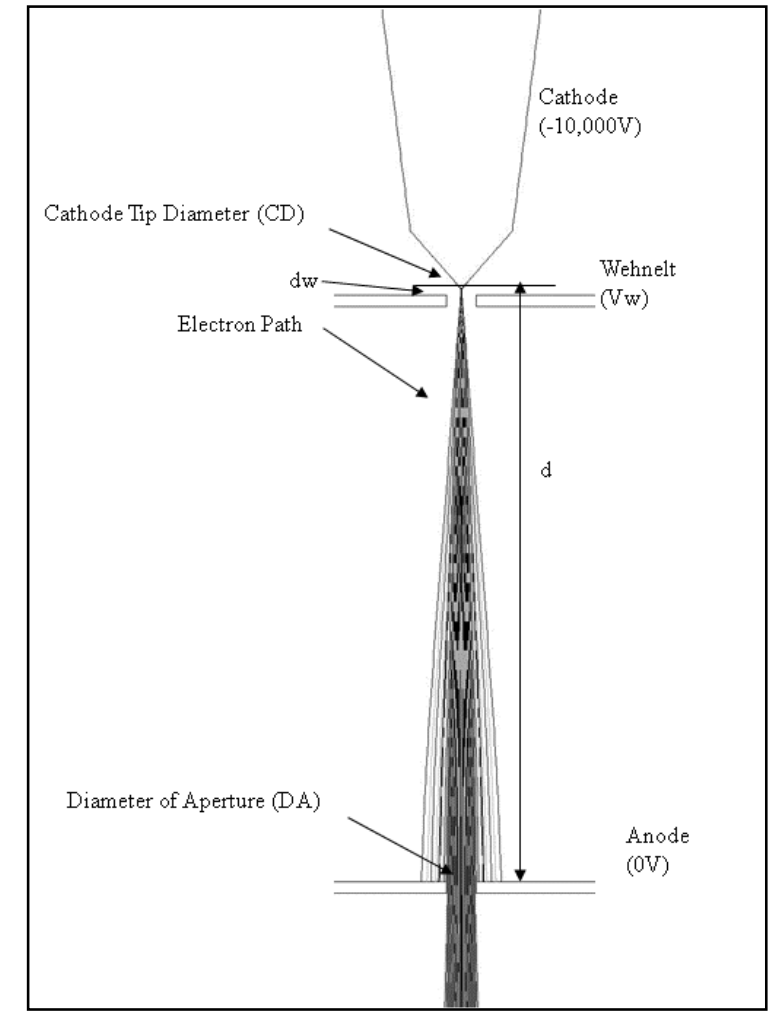

Figure 2. Simulation of the thermionic electron gun in CPO2DS with $V_{w}$ more positive than the cathode.

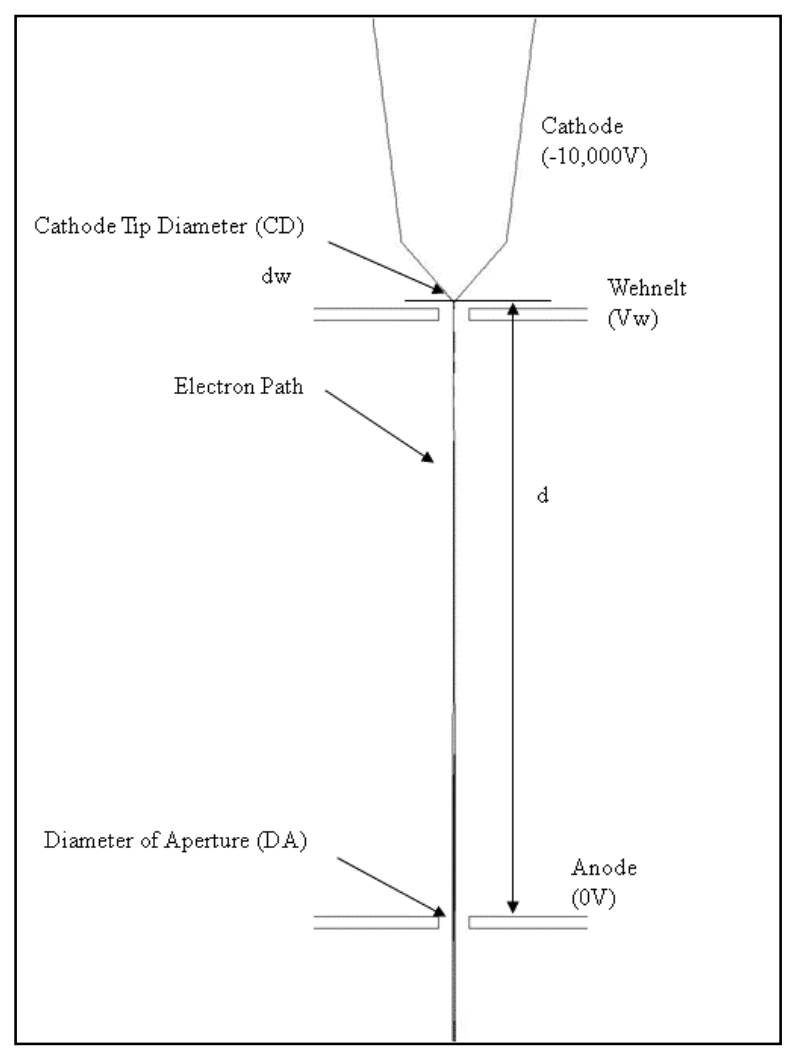

Figure 3. Simulation of the thermionic electron gun in CPO2DS with $V_{w}$ more negative than the cathode.
The effects on the simulated current produced for different voltages on the Wehnelt Cylinder are given below in Table 2 as functions of the two distribution cases. The "equal area" case (which would represent a cold field emitter) and the "equal area" case which represents the thermal emission gun. Table 3 gives the simulated current emitted by the cathode for a range of voltage differences and different temperatures of the cathode. Two different distances between the Wehnelt Cylinder and cathode, $\mathrm{d}_{\mathrm{w}}=0.1 \mathrm{~mm}$ and $0.05 \mathrm{~mm}$, were simulated. Data in the two tables illustrates the Wehnelt Cylinder forcing the current through the anode to be equal to the current emitted by the cathode.

Table 2. Current emitted by the cathode $\left(I_{c}\right)$ and detected by the anode $\left(I_{a}\right)$ as a function of the Wehnelt voltage $\left(V_{w}\right)$ for a fixed cathode voltage of $-10 \mathrm{kV}$ and $d_{w}=1 \mathrm{~mm}$.

\begin{tabular}{|r|r|r|c|l|}
\hline \multicolumn{2}{|l|}{} & \multicolumn{2}{|c|}{ Equal area } & $\begin{array}{l}\text { Equally } \\
\text { spaced }\end{array}$ \\
\hline $\mathrm{V}_{\mathrm{w}}(\mathrm{V})$ & DeltaV & $\mathrm{Ic}(\mu \mathrm{A})$ & \multicolumn{2}{|c|}{$\mathrm{Ia}(\mu \mathrm{A})$} \\
\hline-5000 & 5000 & 9.93 & 5.11 & 5.91 \\
\hline-5500 & 4500 & 9.93 & 5.39 & 6.36 \\
\hline-6000 & 4000 & 9.93 & 5.39 & 6.36 \\
\hline-6500 & 3500 & 9.93 & 5.68 & 6.36 \\
\hline-7000 & 3000 & 9.93 & 5.96 & 6.84 \\
\hline-7500 & 2500 & 9.93 & 6.24 & 7.79 \\
\hline-8000 & 2000 & 9.93 & 7.10 & 7.79 \\
\hline-8500 & 1500 & 9.93 & 8.23 & 8.30 \\
\hline-9000 & 1000 & 9.93 & 9.65 & 9.37 \\
\hline-9500 & 500 & 9.93 & 9.93 & 9.93 \\
\hline-10000 & 0 & 0 & 0 & 0.04 \\
\hline
\end{tabular}

Table 3. Simulated current emitted by cathode for a $d_{w}$ of $0.01 \mathrm{~mm}$ for three different temperatures of the cathode and a range of voltage differences. $N / E$ indicates no emission.

\begin{tabular}{|c|c|c|c|}
\hline $\mathrm{T}(\mathrm{K})$ & 2150 & 2250 & 2350 \\
\hline$\Delta \mathrm{V}(\mathrm{V})$ & \multicolumn{3}{|c|}{$\mathrm{Ic}(\mu \mathrm{A})$} \\
\hline 0 & 2.84 & 9.93 & 21.6 \\
\hline-5 & 2.84 & 9.93 & 15.8 \\
\hline-10 & 2.84 & 8.72 & 10.0 \\
\hline-15 & 2.84 & 5.07 & 5.12 \\
\hline-20 & 1.45 & 1.47 & 1.47 \\
\hline-25 & $\mathrm{~N} / \mathrm{E}$ & N/E & N/E \\
\hline
\end{tabular}

The simulated data for the case in which the distance between the cathode and Wehnelt Cylinder is fixed, for three different cathode temperatures, is shown in Table 4. The voltage difference between the two was varied from 0 to $35 \mathrm{~V}$. When the voltage difference was too large, the current emitted by the cathode was indeed suppressed. 
Table 4. Simulated current emitted by the cathode with $d_{w}=0.05 \mathrm{~mm}$ for three different temperatures of the cathode and a range of voltage differences.

\begin{tabular}{|c|c|c|c|}
\hline $\mathrm{T}(\mathrm{K})$ & 2150 & 2250 & 2350 \\
\hline$\Delta \mathrm{V}(\mathrm{V})$ & \multicolumn{3}{|c|}{$\mathrm{Ic}(\mu \mathrm{A})$} \\
\hline 0 & 2.84 & 9.93 & 32.0 \\
\hline-5 & 2.84 & 9.93 & 29.4 \\
\hline-10 & 2.84 & 9.93 & 24.5 \\
\hline-15 & 2.84 & 9.93 & 18.5 \\
\hline-20 & 2.84 & 9.65 & 12.3 \\
\hline-25 & 2.84 & 6.41 & 6.65 \\
\hline-30 & 2.16 & 2.32 & 2.32 \\
\hline-35 & $\mathrm{~N} / \mathrm{E}$ & $\mathrm{N} / \mathrm{E}$ & $\mathrm{N} / \mathrm{E}$ \\
\hline
\end{tabular}

These simulations showed a strong dependence on temperature and source distribution. The dependence on the voltage difference is seen to be more significant for the configurations with higher currents.

\section{PF Testing and Characterization}

Experimentally, the thermionic gun was powered using the CPS, Inc., commercially available power supply with controls for filament current, accelerating voltage and bias. A separate analog meter monitored the emission current. Figure 4 illustrates the essential elements of the power supply/filament system. $I_{e}$ is the emission current, $R$ is the autobias resistor and $\mathrm{V}_{0}$ is the accelerating voltage. This configuration is of cathode, resistor, and Wehnelt Cylinder is known as self-biasing.

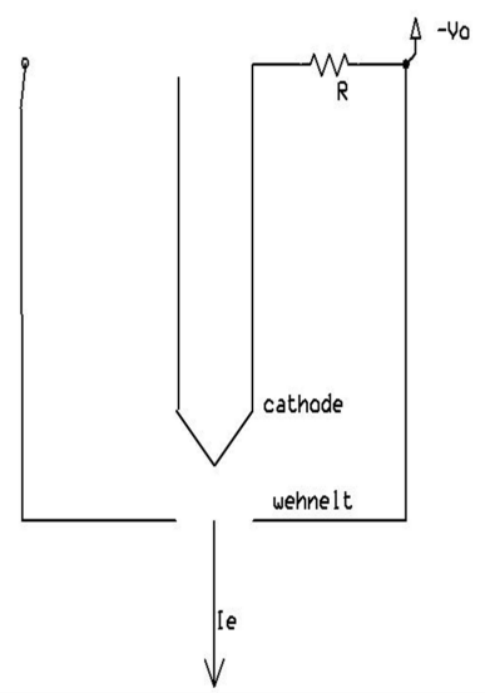

Figure 4. Schematic of cathode filament with Wehnelt Cylinder is shown. The autobias resistor controls the emission current [3], [5].

The autobias resistor controls the emission current by controlling the voltage drop between the Wehnelt Cylinder and the cathode. Since this is self-biasing, as the filament current $\left(\mathrm{I}_{\mathrm{fil}}\right)$ is increased, the emission current increases exponentially until it reaches saturation and becomes reasonably constant. This saturation value strongly depends on the resistance $(\mathrm{R})$ of the autobias resistor.

Experiments were done inside the vacuum sample chamber of an older SEM (Cambridge model 250, mark II). Typical pressures were in the low $10^{-7}$ Torr range. Electrical connections were made through high-voltage and lowvoltage vacuum feedthroughs through a side vacuum flange on the Cambridge sample chamber.

A photo of the in-house constructed Te-gun is given below in Figure 5.

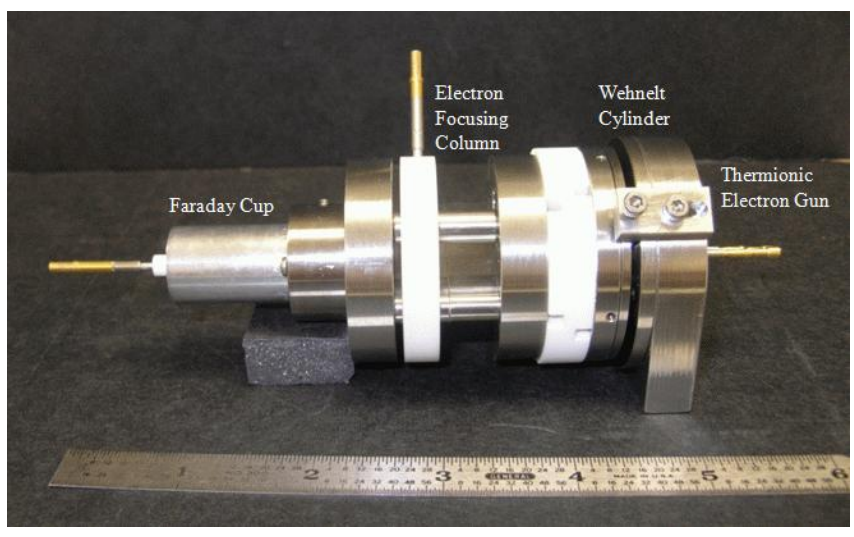

Figure 5. Photograph of the thermionic gun with mSEM assembly.

The figures below plot the experimental results from the thermal emission gun prototype, showing the effect of changing the filament current (Figure 6), the resistance on the autobias resistor (Figure 7) and the accelerating voltage, $\mathrm{V}_{0}$, (Figure 8). Figure 9 shows the current in the Faraday cup as a function of the electron column focusing voltage. As an interesting aside, the experimentally measured current into the Faraday cup as a function of the bias setting was compared to the simulated emission current as a function of temperature, which was an adjustable parameter in the software. This is given in Figure 10. The emission diameter simulated $(0.1 \mu \mathrm{m})$ was chosen so as to produce a current close to that measured experimentally. The error bars are associated with the simulation. The curve shapes are almost identical indicating a linear relationship between the bias and the temperature, at least for the range investigated here. 


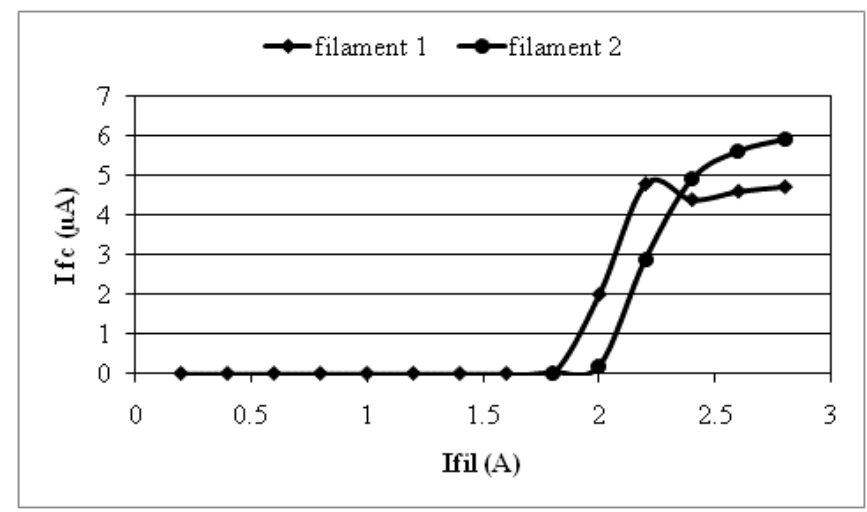

Figure 6. Experimentally measured current in the Faraday cup as a function of current through the filament $\left(I_{\text {fii }}\right)$ for the two different filaments is shown.

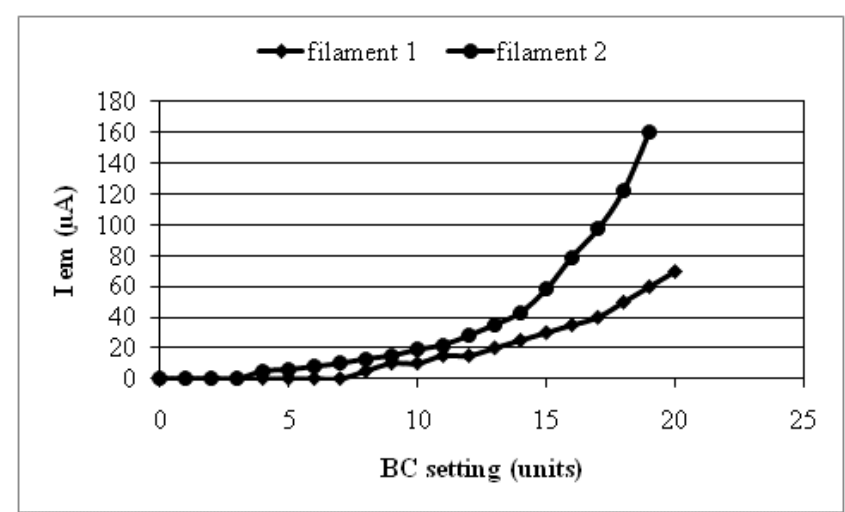

Figure 7. Experimentally measured emission current $\left(I_{e m}\right)$ as a function of the bias control (BC) setting for the two filaments is plotted.

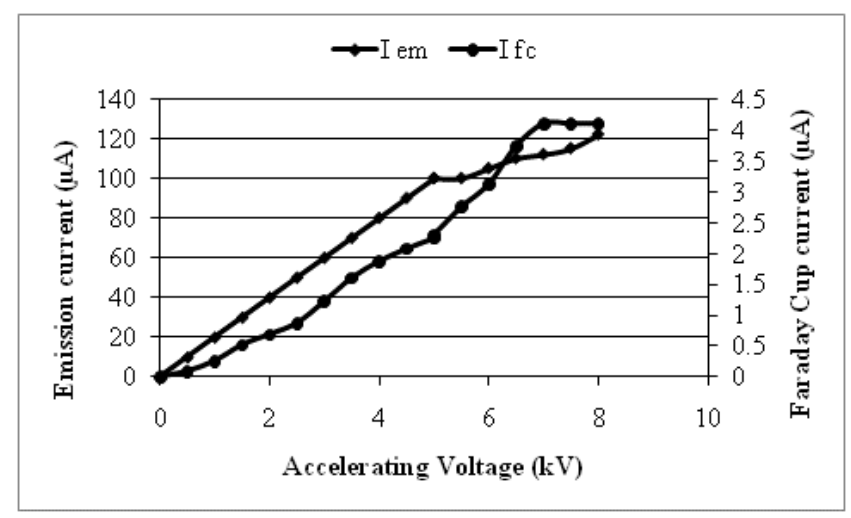

Figure 8. Current in the Faraday cup and emission current as a function of the accelerating voltage is shown.

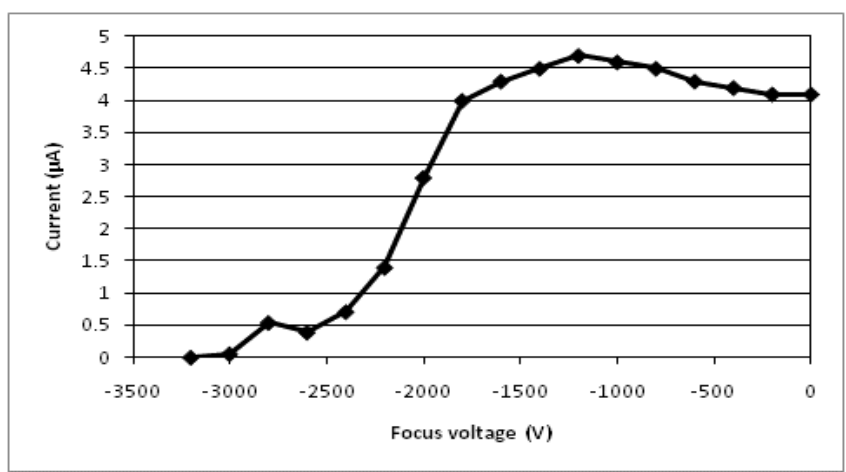

Figure 9. Faraday cup current as a function of electron focusing column - focusing voltage is shown. Optimum operating voltage is around $-2200 \mathrm{~V}$.

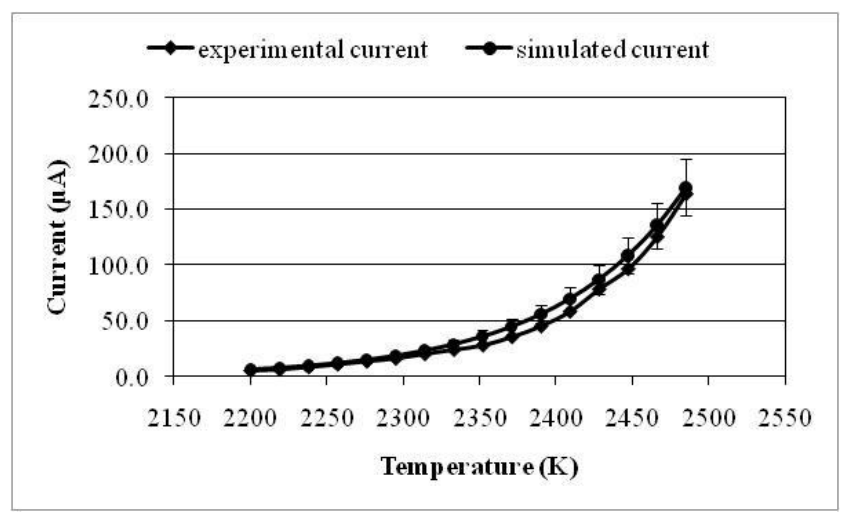

Figure 10. Simulated emission current as a function of temperature is plotted. Experimental results of current into the Faraday cup as a function of bias are also shown and agree well.

In Figure 9, there is a point at which the current measured in the Faraday cup no longer increases (at around -2200V focusing voltage), thus constraining our operating conditions. Figures 6,7 , and 8 are direct characterizations of the filaments we are using and define operational constraints for the power supply that runs them.

\section{COLd Field EMISSION GUN}

The mini-SEM CFEG was fabricated using an off-the-shelf Hitachi tungsten cold field emitter, and is presented in a Butler-like triode configuration [7]. In this configuration, a "sharp" tungsten emitter is followed by a first anode which is at a slightly more positive potential than the emitter, and then by a second grounded anode (Figure 11). 


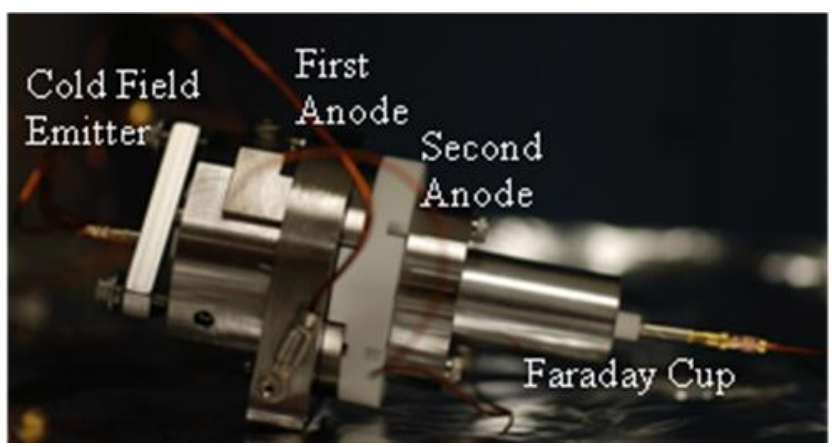

Figure 11. Photograph of the CFEG with Faraday cup attached. The CFE is isolated from the surrounding housing using a MACOR® sleeve.

An emission current results when the potential difference between the tungsten emitter and first anode is large enough to allow electrons to overcome their work function and tunnel out of the tungsten field emitter tip [8]. Successful operation of this CFEG requires one to have fine control over the applied field (and thus, over the emission current) and has necessitated the development of a specialized HighVoltage Power Supply system. Led by our colleagues at $\mathrm{UAH}$, we have designed, fabricated and performed preliminary testing of a novel high-voltage-power supply system to finely control the output emission current from this gun. Described in detail in reference [9], this system allows the user to input a desired emission current and then automatically adjusts the voltage on the first anode to maintain that current. The system regulates off of the combined emitter and first anode currents.

\section{CFEG test \& Characterization}

In order to be able to integrate the CFEG with the remaining components of our mini-SEM, we wanted to first fully characterize the CFEG and High Voltage Power Supply system independently. These tests were carried out in a spherical vacuum chamber which was kept at a pressure of around $\sim 2 \times 10^{-9}$ Torr. Instead of using a sharp tungsten emitter (radius of tens of nanometers), we instead used a highly blunted tip (radius of microns) to carry out this initial testing. During our testing, we have found that a highly blunted tip is much more robust than a sharp tip and can tolerate a higher vacuum. Figure 12 illustrates the difference between the two tips.

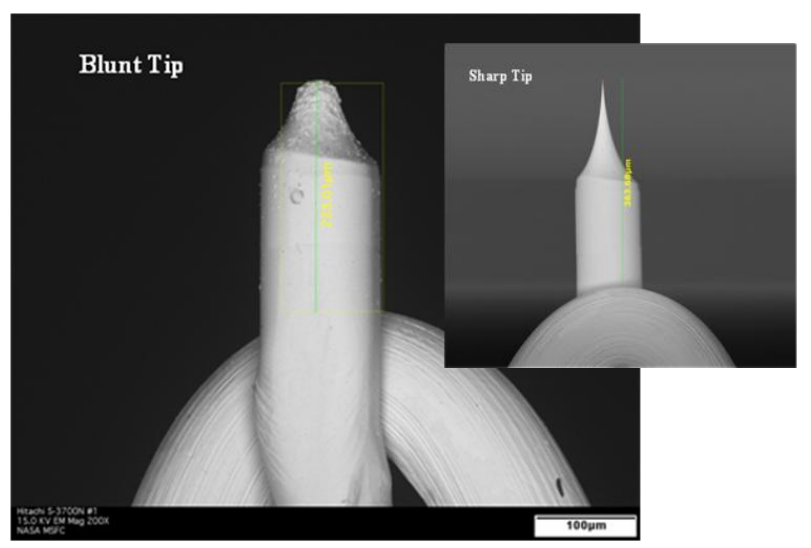

Figure 12. SEM image showing a blunt tip and a sharp tip. The blunt tip is roughly $130 \mu \mathrm{m}$ shorter than the sharp tip and is $\sim 30 \mu \mathrm{m}$ in diameter. Image was taken by G. Jerman (NASA MSFC).

One major drawback to using a blunted tip is that the emission current is not as high as it normally would be when using a sharp tip. Further, the geometry (i.e., the distance between the emitter and extractor) must be modified over that of a sharp tip to produce emission. Despite these drawbacks, the robustness of the blunted emitter makes it ideal to start with, especially since the cost of these highly delicate CFEs is fairly high.

For these tests, a Faraday cup was attached directly to the second anode, such that any current that made it through the second anode (i.e., the current that would be directly inputted into the electron focusing column) would be registered. A portable USB data acquisition systems from IOTech (Personal Daq/3000 Series) was used to record the voltage on the emitter (i.e., the accelerating voltage), the desired input current, the extraction voltage (defined as the voltage difference between the emitter and first anode), and the current registered by the picoammeter that was attached to the Faraday cup. Figure 13 is an image of the test assembly.

Tests were carried out at four different accelerating voltages $\left(A_{V}\right):-4 k V,-6 k V,-8 k V,-10 k V$. For each of these, several desired input currents were entered, ranging from just above $0.400 \mu \mathrm{A}$ to around $1.50 \mu \mathrm{A}$. 


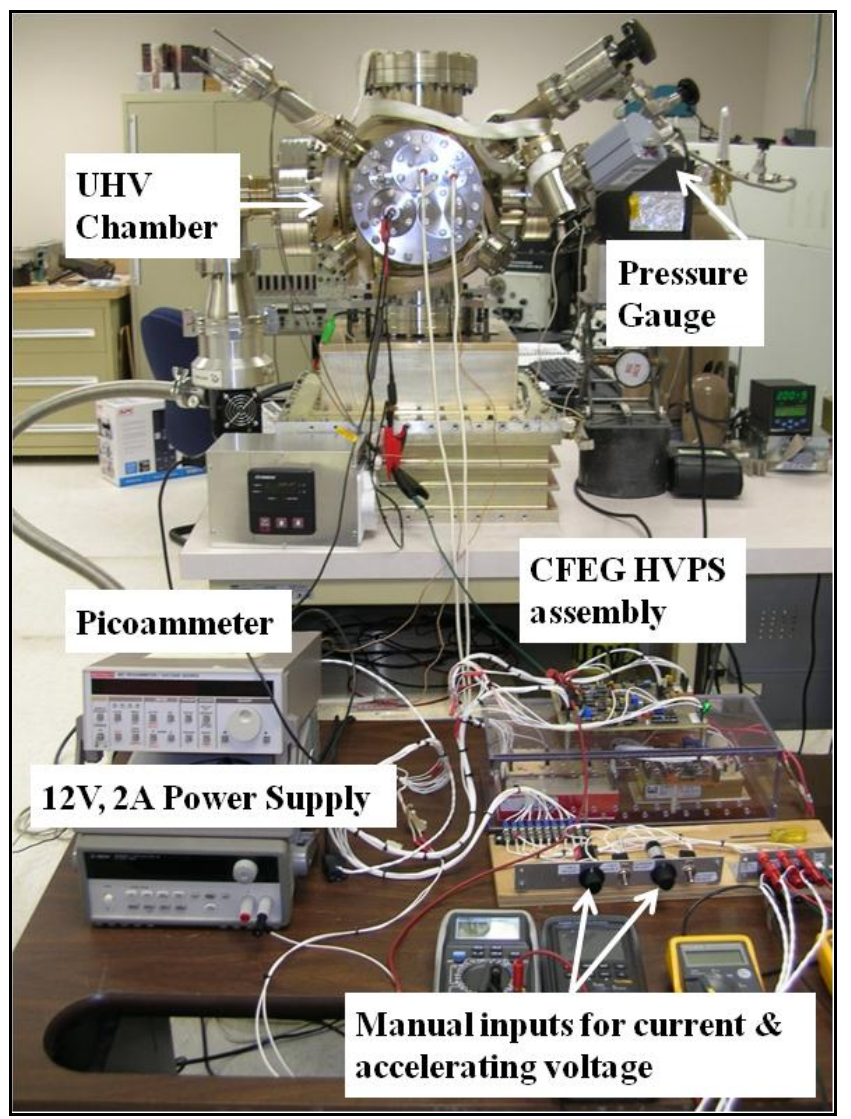

Figure 13. Image of the CFEG test assembly. The accelerating voltage and input current are inputted via two potentiometers. Multimeters were used to monitor real-time response and to verify input and IOTech Daq/3000 (not shown) is used to record data.

\section{CFEG Results \& Discussion}

Figure 14 is a plot of the input current versus the current recorded in the Faraday cup. The error bars are the standard deviation of the mean and are roughly the size of the plot points $\left(\sigma_{X}\right.$ ranges from $0.04 \mathrm{nA}$ to $0.12 \mathrm{nA}$ for the input current and $0.02 \mathrm{nA}$ to $0.92 \mathrm{nA}$ for the Faraday cup current).

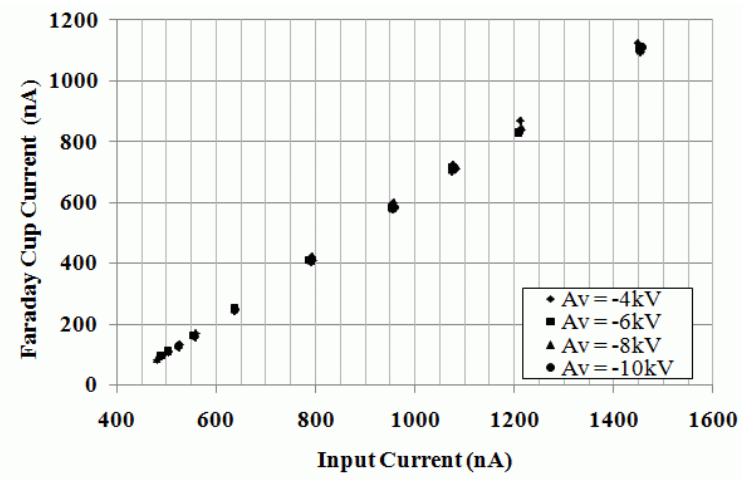

Figure 14. Plot of the input current versus the current measured in the Faraday cup.
From the plot, it is evident that the relationship between the input current and current in the Faraday cup is independent of the accelerating voltage. The current measured in the Faraday cup increases linearly with the input current. A larger fraction of the emission current appears to terminate in the Faraday cup with increasing input current. From simulations, we expected for a large portion of the current being emitted from the cathode to end up on the first and second anodes. Instead, as the input current was increased, we observed the majority of the current in the Faraday cup. Further testing is needed to understand this effect.

Figure 15 is a plot of the extraction voltage (i.e., the voltage difference between the emitter and first anode) as a function of the input current.

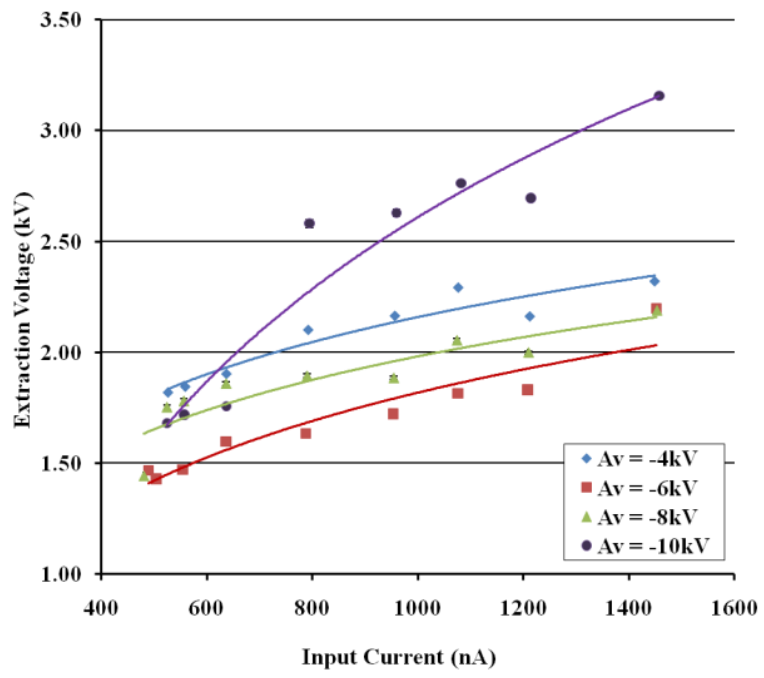

Figure 15. Plot of the input current versus the extraction voltage required to maintain that current, for different accelerating voltages $\left(A_{V}\right)$. The lines are logarithmic fits to the data.

The extraction voltage seems to be very stable (with $\sigma_{X}$ ranging from 0.001 to $0.018 \mathrm{kV}$ ) and increases roughly logarithmically with the input current, deviating slightly from this as the accelerating voltage is increased to $-10 \mathrm{kV}$.

\section{Conclusions}

Two electron guns were constructed; a thermionic pointed filament was assembled and characterized such that the authors could carry out characterization of individual miniSEM components, and a cold field emitter was designed, assembled and characterized for final use with the lunar mini-SEM.

\section{Thermionic Pointed Filament}

The thermal emission gun is of a conventional design and performed as expected using an off-the-shelf power supply. Initial simulations of this gun were performed using CPO2DS software and parameters taken from experimental results. Experimental measurements of the resulting 
emission current were within the range of those resulting from the simulations. Further, the authors were able to identify the optimal operating range for the electron focusing column.

The next stage of testing will be to utilize the secondary electron detector housed inside of the Cambridge SEM sample chamber to obtain an image using this assembly (and constrain the resolution and magnification). Our Co-I, A. Sampson (Advanced Research Systems) is developing a configurable control system for the mini-SEM in the form of an embedded microcontroller. The first priority of this system will be to control the electron beam scanning and image reconstruction. The goal of this work is to be able to operate this mini-SEM without the aid of the Cambridge SEM detectors and imaging system.

\section{Cold Field Emission Gun}

It is clear from our first round of data that we have only just begun to characterize the CFEG. The linear response and high percentage of the emission current that is registered in the Faraday cup is encouraging. However, there should be a point at which we should see the current in the Faraday cup flatten out, as we approach the maximum allowable emission current for this emitter. Despite the fact that we haven't seen this, the High Voltage Power Supply assembly seems to be regulating the desired current in a very stable way.

Near-term plans are to image the emission spot just after the second anode using a phosphor screen followed by a glass viewport and CCD camera. This set-up is similar to that of Yeong and Thong [10], in which they recorded the life-cycle of a CFE. Imaging the spot after the second anode will allow us to verify our previous results and will give us an idea of what the emission from such a highly blunted emitter will look like. Following the success of these tests, we plan on replacing the blunt tip with a shaped, sharp tip to better understand our system response under these more desirable conditions. As the sharp tip will require a more stringent vacuum system, we plan on installing three non-evaporable getters (NEGs) to act as an additional pumping system.

Subsequent to the successful outcome of the CFEG sharpemitter testing, we plan on integrating the CFEG with our electron focusing column and scanning/imaging system and backscatter detector, for a complete stand-alone system.

\section{REFERENCES}

[1] K. G. Thaisen, et al., "An ESEM/SEM Study of Lunar Soil and the Potential for a Miniaturized Version on the Moon," 40 ${ }^{\text {th }}$ LPSC Conf., March 23-27, 2009.

[2] K. G. Thaisen, et al., Development of a Miniature Scanning Electron Microscope to Facilitate In Situ Lunar Science and Engineering," Proceedings of the $12^{\text {th }}$ International Conference on Engineering, Science, Construction, and Operations in Challenging Environments, March 14-17 ${ }^{\text {th }}$, 1046, 2010.

[3] Joseph Goldstein, et al., Scanning Electron Microscopy and X-Ray Microanalysis, New York: Springer Science and Business Media, LLC, 2003.

[4] EBSciences Web site http://www.ebsstore.com

[5] D.B. Williams and C.B. Carter, Transmission Electron Microscopy: a textbook for material science, Imaging Part 3, Springer: New York, 2009.

[6] D. Manura, Charged Particle Optics software, computer software, Manchester, UK, CPO Ltd., 2008. The software is available through Scientific Instrument Services, Inc. (http://www.sisweb.com).

[7] T. W. Butler, "Digital Computer Techniques in Electron Microscopy," 6th International Congress Electron Microscopy (Kyoto) 1, 193, 1966.

[8] R.H. Fowler and L. Nordheim, "Electron Emission in Intense Electric Fields," Proceedings of the Royal Society of London, 119 (781), 173-181, 1928.

[9] J. Gaskin et al., "Miniaturized Scanning Electron Microscope for In-Situ Planetary Studies," Proceedings of the $12^{\text {th }}$ International Conference on Engineering, Science, Construction, and Operations in Challenging Environments, March 14-17 ${ }^{\text {th }}, 2010$.

[10] K. S. Yeong and T. L. Thong, "Life Cycle of a Tungsten Cold Field Emitter," Journal of Applied Physics, 99, 104903, 2006. 


\section{BIOGRAPHIES}

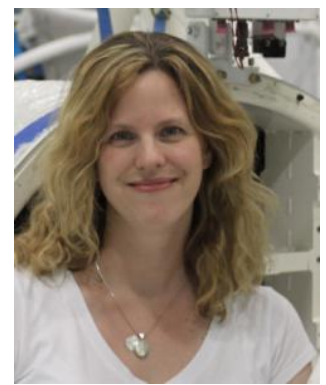

Dr. Jessica Gaskin is a physicist in the X-Ray Astronomy Group at NASA Marshall Space Flight Center in Huntsville, AL. She has characterized multiple types of solid state detector systems for X-Ray Astronomy including many-pixel Cadmium-Zinc-Telluride detectors and Silicon Drift Detectors. She also supports the High Energy replicated Optics (HERO) hard X-ray balloon-borne telescope; offering flight support and detector calibration support. Over the past few years, she has focused her research on Planetary/In-Situ based instrumentation, concentrating on the mini-SEM. She has a B.S. in Physics (specializing in Astrophysics) from New Mexico Tech, an M.S. in Astronomy from Case Western Reserve University, and a Ph.D. in Physics from the University of Alabama in Huntsville.

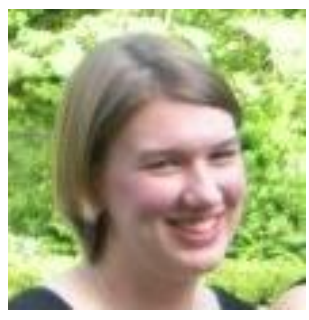

Stephanie Medley is a graduate student at University of Alabama in Huntsville. She received her Bachelor's in Physics and Mathematics in 2009 from UAH. Including graduating magna cum laude, she received several academic awards while achieving her undergraduate degree. She was also accepted into the Sigma Pi Sigma Physics Honor Society. She was involved in the research experience for undergraduates at UAH. She has been involved in several scientific organizations, including SPIE, the International Society for Optical Engineers, SPS, the Society of Physics Students.

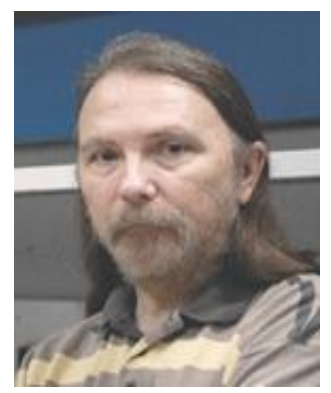

Dr. Don Gregory is professor of physics a the University of Alabama in Huntsville and has more than 100 refereed open literature technical publications in internationally circulated journals in fields ranging from basic physics and optics to materials and advanced propulsion and new energy sources. He has 13 US patents and has been the recipient of the Department of the Army Research and Development Award, the Department of the Army Technology Transfer Award and the IEEE Fink Award. He has graduated 19 Ph.D. students and 20 M.S. students in disciplines within science and engineering. He has also published two commercially successful books on World War II history.

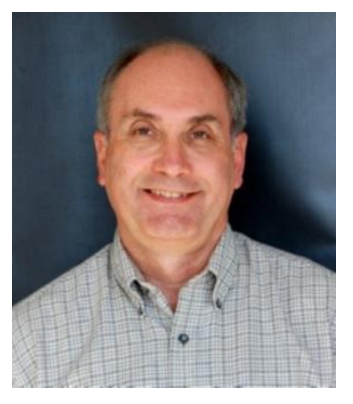

Terry Abbott is an electrical engineer working in the Rotorcraft Systems Engineering and Simulation Center at the University of Alabama in Huntsville. He has worked as a product design engineer and a test engineer in his career at Reliance Electric, GTE, Chrysler Corporation and Siemens Corp. Most recently he was a Test Engineering Manager at the Siemens Automotive Electronics Division in Huntsville, Alabama. He has a BEE from Cleveland State University and a master's degree in business from Auburn University.

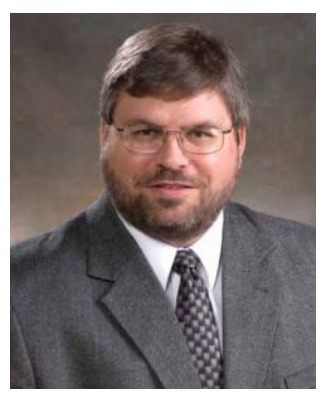

Gregory Jerman is a materials scientist working in the Failure Analysis and Metallurgy Branch at NASA Marshall Space Flight Center in Huntsville, AL. He has over 20 years experience in the application and operation of electron microscopy equipment including environmental SEM, field emission SEM, TEM, and electron microprobe. His applied background includes failure analysis of metallic and nonmetallic aerospace materials and more than a decade of astrobiology experience with the analysis of meteorites, terrestrial rocks, fossilized bacteria, and living microorganisms. He has a B.S. in physics from Rensselaer Polytechnic Institute and a M.S. in metallurgy from Iowa State University.

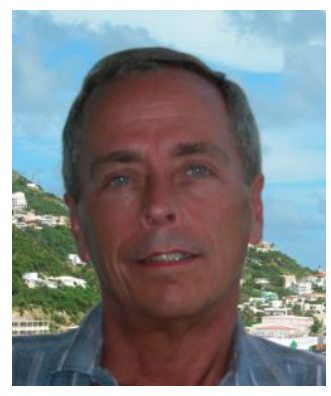

Allen R. Sampson has been self employed in electron microscopy and analytical instrumentation consultation and contracting for 30 years. During this time he has assisted research in a wide variety of disciplines for government, academic and commercial customers. He has produced a number of hardware and software solutions for his customers in automation, image processing and quantitative analysis and carried on independent research in computer science and electrochemistry. He previously worked for IBM, Exxon and Standard Oil of Indiana, where he designed the first general purpose computer database system and the concept of a graphical computer kiosk in the early 70's.

\section{ACKNOWLEDGMENTS}

The authors would like to thank the Planetary Instrument Definition and Development Program, offered out of the NASA NSPIRES Research Opportunities in Space and Earth Science (ROSES) program, for funding this development. 